\title{
Ex-Vivo and In-Vivo Studies in a Porcine Model for Experimental Validation of an Embedded System Designed for Radiofrequency Ablation of Hepatocellular Carcinoma
}

\author{
O. F. Gaidos ${ }^{1}$, M. P. Marques ${ }^{2}$, J. Y. Ishihara ${ }^{1}$, S. R. F. Rosa ${ }^{2}$ \\ ${ }^{1}$ Postgraduate Program in Electronic and Automation Systems Engineering, University of Brasilia \\ Darcy Riveiro Campus, Brasilia D.F., Brasil \\ ogaidos@gmail.com; ishihara@ene.unb.br \\ ${ }^{2}$ Bioengineering Group, Postgraduate Program in Biomedical Engineering, University of Brasilia \\ Gama Faculty, Brasilia, Brasil \\ marina.pin.mar@gmail.com; suelia@unb.br
}

\begin{abstract}
The Hepatocellular Carcinoma is the main cause of morbidity and mortality in the world related to primary liver cancer. One of the principal treatment for hepatocellular cancer and effective clinical outcomes is Radiofrequency Ablation (RFA). In Brazil, the commercial equipments used in RFA are expensive and it isn't open technology. One problem during the RFA procedure is the thermal lesion volume estimation because parameters such as temperature or impedance are not fully controlled in the ablation zone, generating an uncertainty in the surgical procedure. This paper presents prototype validation based on experimental ablation protocol applied in exvivo and in-vivo tests on porcine liver of an RFA embedded system for the treatment of primary liver cancer, based on experimental results, calculating area and volume in the ablation zone. Using the signals of temperature and bioimpedance on porcine liver and the data processing in a microcontroller, it controls the power of a custom radiofrequency generator designed up to $50 \mathrm{~W}$ and frequency of $400 \mathrm{kHz}$, this energy is applied across an umbrella-shaped RF electrode. The data are sent by RS232 protocol, showed on LCD screen and sent to a computer for later analysis. Finally, it was possible to validate a hardware prototype with low-cost and low-power applied to thermal ablation of liver tumors.
\end{abstract}

Keywords: Size ablation, ATMEGA128 Microcontroller, Radiofrequency Generator, Liver Porcine, Experimental Protocol.

\section{Introduction}

The present work analyzes experimental outcomes in a porcine model using an embedded system prototype for the thermal treatment of liver cancer, the thermal procedure is called RFA, it's the burn of unwanted cells in the liver through the application of electromagnetic waves of RF. The thermodynamics of RF tissue heating, the electrical conductivity of tissue and its change with temperature are discussed in [1]. The state of art of RF ablation technique is presented in [2]. The ex-vivo validation for impedance controlled RF tumor ablation and Mathematical model can be found in [3]. The safety of electrosurgical units for both bipolar and monopolar electrosurgery were approach in [4]. The examination of the patterns of all injuries and deaths related to electrosurgical devices reported to the Food and Drug Administration (FDA), they are shown in [5]. Ablation techniques for primary and metastatic liver tumors are described in [6]. Comparisons of efficacy ablation lesions between magnetically coupled bipolar electrodes and conventional monopolar and bipolar RFA electrodes, were made in [7]. Size and geometry of the ablation zone obtained by RF electrodes are highly variable. The development of a bipolar 2 x 2 electrode system, with ex-vivo liver experiments and finite element method modeling can be found in [8]. The in-vivo experiments and computational simulations were realized showing that the thermal lesion shape created around the portal vein was significantly modified by heat sink effect [9]. Ex-vivo experiments with bovine liver were realized for displacement of roll-off (phenomenon occurs by tissue carbonization, it decreases the current flow and consequently it's a limitation for increase temperature) with aim of increase the ablation zone, a dry RF electrode together with customized cooling system where several cool liquids were used, the best result was using deionized water at $5^{\circ} \mathrm{C}$, in this case the ablation size increase up to 2.5 times respected to dry electrode (not use cool liquid), the results are shown in [10]. The RFA is a therapy for tumors minimally invasive. In this treatment, the tumor is diagnosed and located by image guidance, the RF electrode is inserted inside the tumor and the tissue around the electrode is heated using a RF generator. The goal is to kill 
the tumor with a minimal damage to surrounding healthy tissue. Today, although this intervention is realized in clinical practice, the outcome is difficult to predict and satisfactory outcomes depend of different factors such as tumor location, the presence of large blood vessels in close proximity to the tumor or the output power control of RF generator. The exact area of necrosis extension is difficult to plan and control. Some common problems in the electrosurgery are the burns and injuries caused for excess power or high density of current in unexpected locations such as back and leg of the patient, because of poorly contact between patient and grounding pad. Several researches about RFA are based on commercial models of medical devices, but for the local development of biomedical instrumentation and new electromedical equipments in Brazil, it is necessary to develop national products in this area. We propose to analyze ex-vivo and in-vivo experiments in pig liver for the process validation related to our RFA prototype, the understand of these outcomes could give an alternative solution for the problems of hepatic RFA with the intention to increase the safety and effectiveness of the equipment, in addition to reducing costs for this equipment because in the Brazilian electromedical equipments market, this device is imported. We contributions are related to validate an ARF prototype using ex-vivo and in-vivo experiments in a porcine model with several experimental protocol ablation and their relation with size ablation, also it is presents the setup measuring of temperature for RF electrodes without cooling system.

\section{Hardware}

This section describes the hardware architecture for the RFA system developed in the Bioengineering Group, Postgraduate Program of Biomedical Engineering located at the University of Brasilia (UnB). The most important signals in RFA are the impedance and temperature in the ablation zone and the power released by RF generator, this component was designed for an output power up to $50 \mathrm{~W}$ and frequency of $400 \mathrm{kHz}$. The Fig. 1 shows the prototype diagram, the first step in the RFA treatment is to place RF electrode into patient's liver, for this approach is used image-guided thermal ablation such as Ultrasound (US), Computed Tomography (TC) or Magnetic Resonance Imaging (MRI), for this research we don't implement this stage. A umbrella-shaped RF electrode was used for to apply RF energy, it's manufactured by Boston Scientific (model 4.0 (M001262240) LeVeen CoAccess Needle Electrode System) of $4 \mathrm{~cm}$ in diameter, cannula length of 15 $\mathrm{cm}$, this type of electrode is dry (non-cooling system) and it has not temperature sensor integrated in the tips of electrode, for this reason we design and develop a customized temperature sensor (see Fig. 3 (left)). The second step is turn on the RF Generator in low power and then to increase power up to maximum level and consequently the tissue is heating, when value of temperature reaches $60^{\circ} \mathrm{C}$ is guaranteed necrosis tissue (dead cells). The Bioimpedance signal is important because this parameter is related to hepatocytes (liver's cells), when cell dead occur via high temperatures, the impedance increase abruptly. The temperature data in the ablation zone, power of RF generator and impedance of liver are processed in an embedded system (ATMEGA128 microcontroller manufactured by ATMEL company), this component has an Inter Integrated Circuit (I2C) interface for the communication with a real time clock (DS1307), providing information of hours, minutes and seconds related to the surgical time. Also through Serial Peripheral Interface (SPI) is realized the communication with the MAX6675 temperature sensor with K-type thermocouple. According to this, the power of RF generator is controlled based on different experimental protocol and the energy is applied to an umbrella-shaped RF electrode into liver malignant tumor. Furthermore, the data are showed in a LCD (display) for monitoring the electrical and physical variables. See Fig. 2. Finally the data are send to computer for later analysis. 


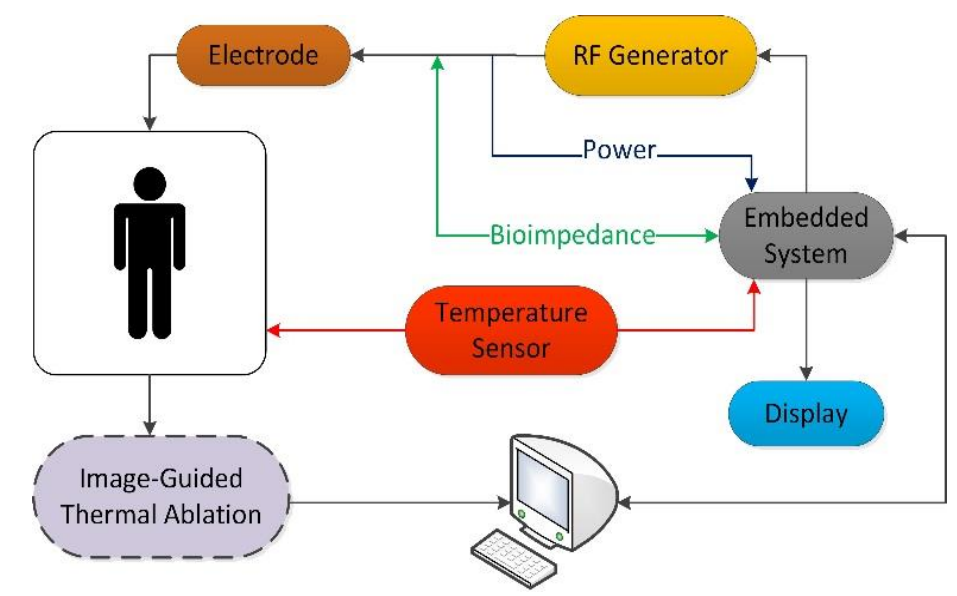

Fig. 1: Block diagram of RFA unit. Steps for detection and treatment in liver Cancer: a) Placement of RF electrode using image-guided thermal ablation as Ultrasound Machine, Computed Tomography or Magnetic Resonance (purple block). The dashed line meaning that block isn't implement in this work. b) Continuous monitoring and processing of physical and electrical variables (Temperature sensor (red block), impedance signal in the body (green line) and output power signal (blue line)). The embedded system (gray block) active the RF generator (orange block) using the power necessary for it be applied using an RF electrode (brown block) in the local tumor

(liver's patient). Finally the data are send to computer for later analysis.
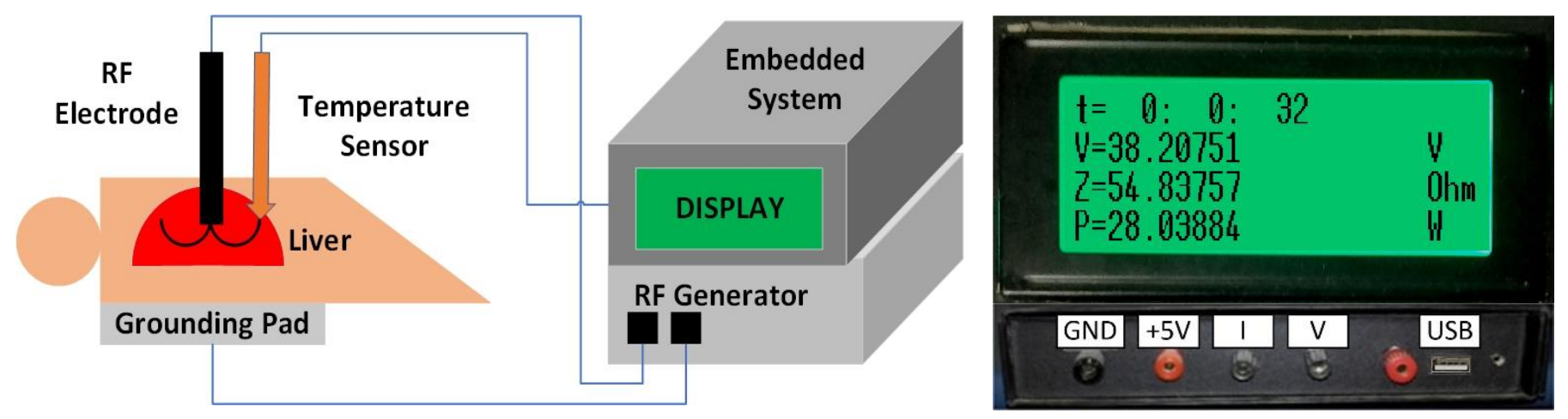

Fig. 2: Setup Diagram for in-vivo experiments showing the patient's liver, the RF electrode connected to RF Generator, temperature sensor connected to Embedded System and grounding pad connected to RF Generator (left). Hardware Prototype (right), containing a LCD screen for visualization of data. The time " $\mathrm{t}$ " is measured in hours, minutes and seconds, the voltage "V" is measured in volts, the impedance " $\mathrm{Z}$ " is measured in Ohms, the power "P" is measured in watts, the temperature "T" (not showed in LCD) is measured in

${ }^{\circ} \mathrm{C}$, the current "I" (not showed in LCD is measured in Amperes). The output connectors called "I" and "V" are used to measure current and voltage in the electrical circuit, " 5 " is the power signal of $5 \mathrm{~V}$ and "GND" is the grounding signal. USB connector is used for communication between prototype and computer sending data for later analysis.

\section{Ex-vivo Experiments}

The present study was based on ex-vivo experiments using porcine liver, evaluating the temperature in the ablation zone and applying a RF of $400 \mathrm{kHz}$ with a power generator setting of $50 \mathrm{~W}$. Freshly extracted porcine livers were obtained from a local slaughterhouse. Before starting the experiments, the tissue remained at a temperature of $8{ }^{\circ} \mathrm{C}$ approximately. A Boston $\mathrm{RF}$ electrode was used in the procedure, it was inserted $2 \mathrm{~cm}$ inside the liver. For the measure of temperature used in the ablation zone was used the MAX6675 temperature sensor placement $2 \mathrm{~cm}$ related to Boston RF electrode. For grounding pad was used a dispersive metallic electrode $\left(180 \times 300 \mathrm{~mm}^{2}\right)$. See Fig. 3. The zones of thermal lesions were choosing randomly between the 5 pig liver lobules. For whole experiments, the RF generator was configured with initial voltage of 10 $\mathrm{V}$ and it was increased gradually for $2 \mathrm{~V}$ each $60 \mathrm{~s}$ up to $50 \mathrm{~W}$. The ablation time was 15 minutes. 


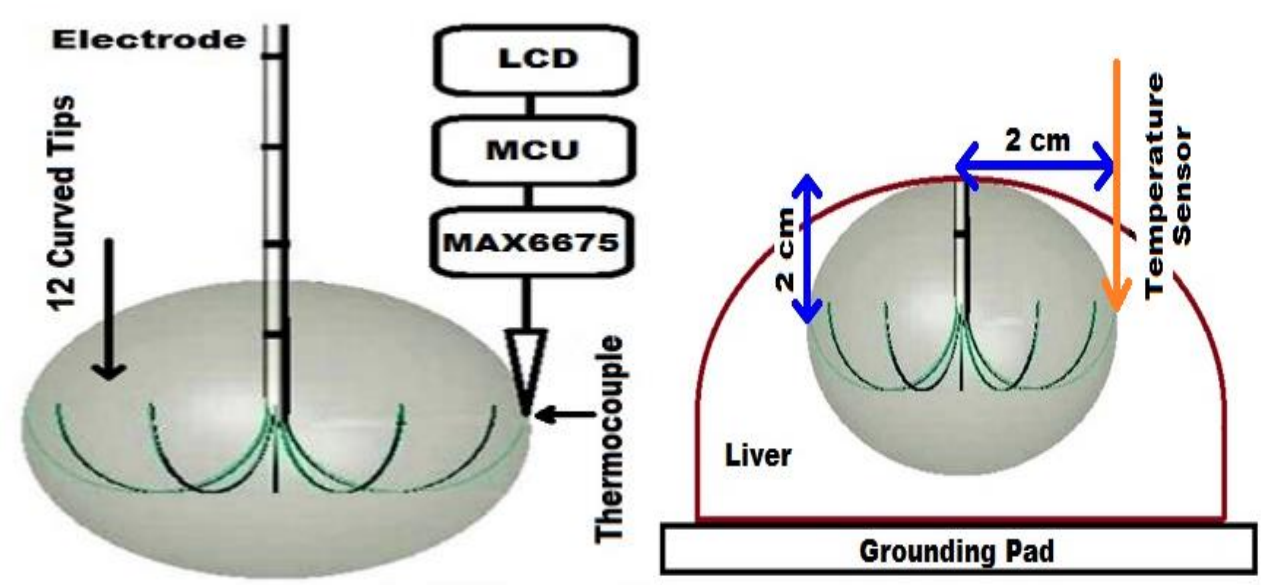

Fig. 3: Setup Measuring related to retractable curved tip of Boston RF electrode and position of a customized temperature sensor, this component is done of K-type thermocouple, integrated circuit MAX6675, ATMEGA128 microcontroller (MCU) and LCD (left). Setup placement of Boston RF electrode, temperature sensor and grounding pad on the pig liver (right). Source: Adapted from [11].

\section{In-vivo Experiments}

In the Surgical Center, Department of Medicine in PUC (Pontifice Catholic University) of Goias, under technic supervision of teacher Physician Fátima Mrué, whole procedures realized occurs with observance of governed precepts by Ethical commission guideline for animal use (CEUA) - animals of surgical practice. The in-vivo experiments were realized using 5 female pigs subjected to abdominal surgery by median laparotomy. The Boston RF electrode was inserted $2 \mathrm{~cm}$ inside the liver. The distance between Boston RF electrode and temperature sensor was $2 \mathrm{~cm}$. See Fig. 3 .

The animals were divided in "ablation tests", where we have 5 animals randomly distributed. The ablation tests were called RFA1, RFA2, RFA3, RFA4, RFA5, RFA6, RFA7 related to the Boston RF electrode setup and temperature sensor (percutaneous (lateral incision) or perpendicular (to the exposed liver)). See Fig. 4. The Table I summarizes the experimental ablation protocol.

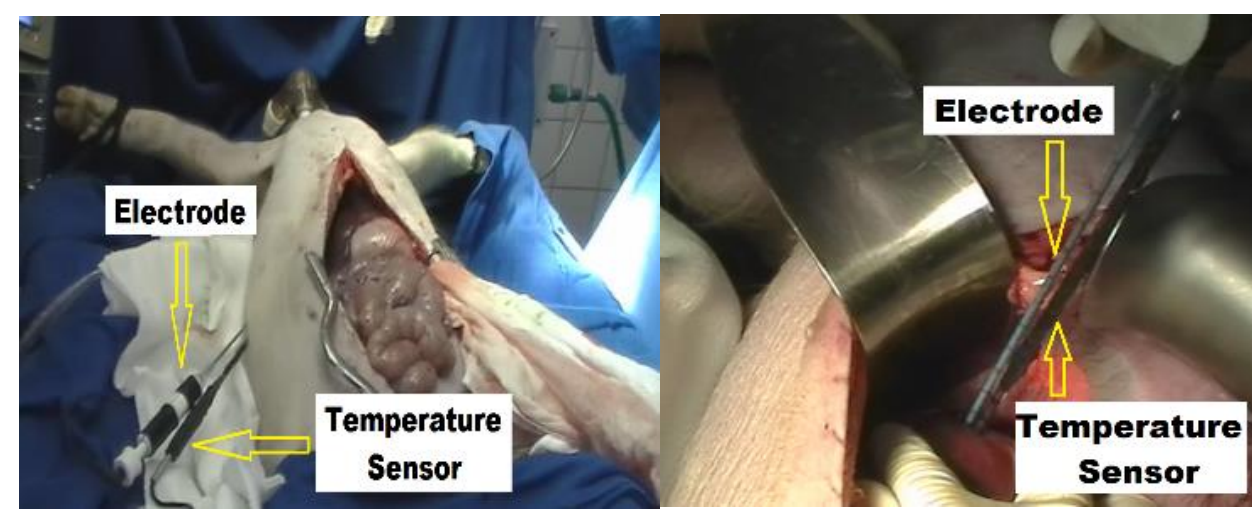

Fig. 4: In-vivo experiments with percutaneous setup (lateral incision) of Boston RF electrode and temperature sensor (left). Perpendicular setup (to the exposed liver) of Boston RF electrode and temperature sensor on ablation zone (right). 
Table 1: Experimental ablation protocol for in-vivo experiments according to location of Boston RF electrode, temperature sensor and ablation time.

\begin{tabular}{|c|c|c|c|c|c|}
\hline Pig & Test & $\begin{array}{c}\text { Location of } \\
\text { RF Electrode/ } \\
\text { Temperature Sensor }\end{array}$ & $\begin{array}{c}\text { Initial/ } \\
\text { Step/ } \\
\text { Final Voltage (V) }\end{array}$ & $\begin{array}{c}\text { Voltage } \\
\text { Increase } \\
\text { with time (s) }\end{array}$ & $\begin{array}{c}\text { Total } \\
\text { Ablation } \\
\text { Time (min) }\end{array}$ \\
\hline 1 & RFA1 & $\begin{array}{c}\text { Perpendicular/ } \\
\text { Perpendicular }\end{array}$ & $10 / 2 / 28$ & 120 & 12 \\
\hline 2 & RFA2 & $\begin{array}{c}\text { Percutaneous/ } \\
\text { Percutaneous }\end{array}$ & $22 /-/ 22$ & - & 15 \\
\hline 3 & RFA3 & $\begin{array}{c}\text { Perpendicular/ } \\
\text { Perpendicular }\end{array}$ & $10 / 2 / 29.5$ & 60 & 14 \\
\hline 4 & RFA4 & $\begin{array}{c}\text { Percutaneous/ } \\
\text { Perpendicular }\end{array}$ & $10 / 2 / 26.5$ & 60 & 14.41 \\
\hline 5 & RFA5 & $\begin{array}{c}\text { Perpendicular/ } \\
\text { Perpendicular }\end{array}$ & $50 /-/ 50$ & - & 19.44 \\
\hline 5 & RFA6 & $\begin{array}{c}\text { Percutaneous/ } \\
\text { Percutaneous }\end{array}$ & $10 / 2 / 24$ & 30 & 10.8 \\
\hline 5 & RFA7 & $\begin{array}{c}\text { Perpendicular/ } \\
\text { Perpendicular }\end{array}$ & $10 / 2 / 43.3$ & 30 & 15.6 \\
\hline
\end{tabular}

The Table I presents the experimental ablation protocol for in-vivo experiments. In this case, we use 5 female pigs for 7 different test called RFA1 up to RFA7. Each test has different setup related to initial voltage ablation, step voltage (for example for RFA1 test, the step voltage is increase $2 \mathrm{~V}$ each $120 \mathrm{~s}$ ), final voltage ( $28 \mathrm{~V}$ for RFA1 test). Finally is showed the total time ablation for each test (12 minutes for RFA1 test). Other setup is Boston RF electrode location (perpendicular (to the exposed liver) or percutaneous (lateral incision)) and temperature sensor location (perpendicular or percutaneous).

\section{Results}

This section shows the power, impedance and temperature measures and relation with size ablation depending of used protocol ablation. These outcomes were realized using ex-vivo and in-vivo experiments.

\subsection{Power Measurement}

The Power is supplied by a custom RF Generator developed in our laboratory. The power curve for RFA1 protocol is showed in Fig. 5 (top-left), where approximately in $500 \mathrm{~s}$, decrease the value of power because in this time, take place the dead cell, and consequently vaporization of tissue, that cause non-conduction of electricity. 


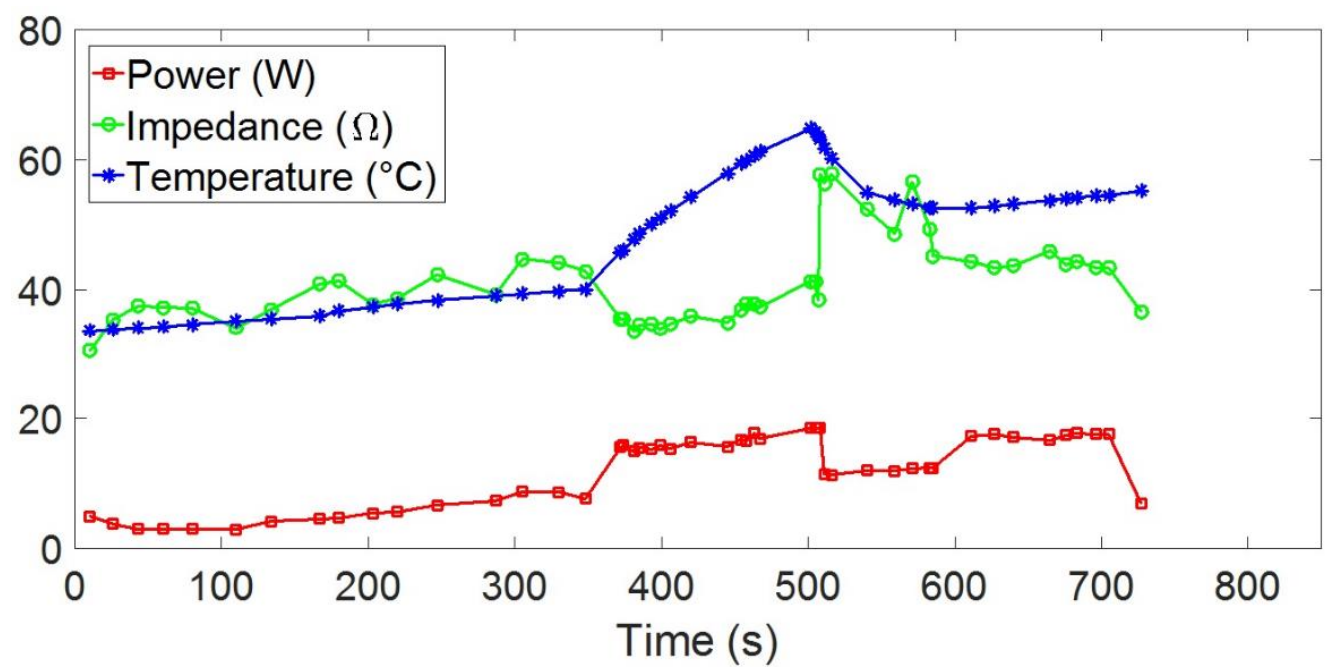

Fig. 5: Measured Power at the interior of tissue during 12 minutes for RFA1 protocol in in-vivo experiments (top-left). Measured Impedance at the interior of tissue during 12 minutes for RFA1 protocol in in-vivo experiments. In $500 \mathrm{~s}$, the value of impedance increase abruptly, known as the "roll-off" phenomenon, this topic is discussed in [1] (top-right). Measured Temperature at the interior of tissue during 12 minutes for RFA1 protocol in in-vivo experiments. Approximately in $500 \mathrm{~s}$, the temperature reaches $60{ }^{\circ} \mathrm{C}$, where cell dead occur (necrosis tissue) (bottom-right). Impedance (red line), Power (green line) and Temperature (blue line) simultaneously (bottom-left).

\subsection{Bioimpedance Measurement}

The Bioimpedance curve for RFA1 test is showed in Fig. 5 (top-right), where approximately in $500 \mathrm{~s}$, the value of impedance increase abruptly, known as the "roll-off" phenomenon (as discussed in [10]), this event is associated to cell dead, in the other words necrosis tissue.

\subsection{Temperature Measurement}

The temperature curve is showed in Fig. 5 (bottom), where approximately in $500 \mathrm{~s}$, the value of temperature decrease, because for temperatures greater than $60{ }^{\circ} \mathrm{C}$, cell dead occur (necrosis tissue), then the vaporized or carbonized tissue around the Boston RF electrode operates as an insulator which prevents extension of the ablation region, decreasing the temperature in the ablation zone.

Following the experimental procedure based on Section III (Ex-Vivo Test), different ex-vivo experiments were realized to validate the prototype.

For 5 liver samples, the lesion size was approximated by an ellipse, calculating the major diameter, minor diameter and area, in this case, we use the ImageJ $1.50 \mathrm{i}$ software, which is an image processing program developed at the National Institute of Health (NIH). To calculate the volume of the thermal lesion, it was approximate to a spheroid volume (as in (1)):

$$
V=\left(\frac{\pi}{6}\right) *\left(A^{2}\right) * C
$$

Where $\mathrm{A}$ is the major diameter and $\mathrm{C}$ is the minor diameter. See Fig. 6. The result can be seen in Table 2. The theoretical volume based on Boston RF electrode Geometry [11] is:

$$
V_{\text {theoretical }}=\left(\frac{\pi}{6}\right) *\left(4^{2}\right) * 3.3=27.65 \mathrm{~cm}^{3}
$$




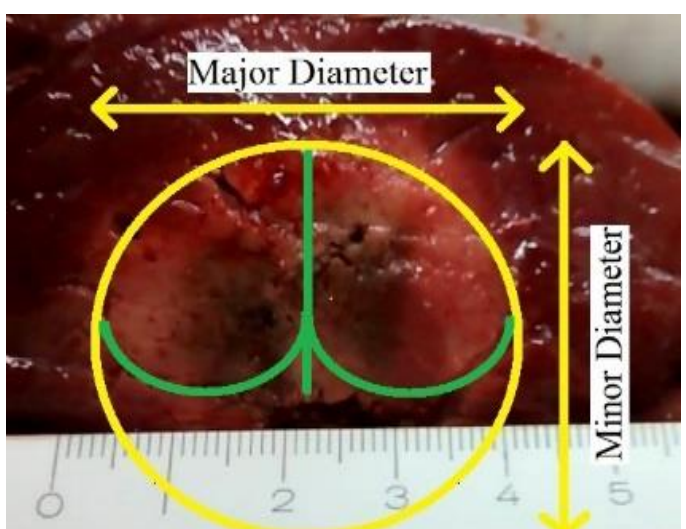

Fig. 6: Measurement setup for RFA procedure using ImageJ software. Measurement setup for ablation zone showing the major and minor diameter based on ellipse (right). The placement of Boston RF electrode is represented by green line.

Table 2: The mean diameters and volumes of the ablated zones according to sample of liver in ex-vivo experiments using Boston RF electrode of $4 \mathrm{~cm}$ in diameter.

\begin{tabular}{|c|c|c|c|c|c|}
\hline \multirow{2}{*}{ Measures } & \multicolumn{5}{|c|}{ Liver Samples } \\
\cline { 2 - 6 } & 1 & 2 & 3 & 4 & 5 \\
\hline Major $(\mathrm{cm})$ & $2.776 \pm 0.5$ & $3.7 \pm 0.5$ & $3.83 \pm 0.5$ & $3.87 \pm 0.5$ & $4.34 \pm 0.5$ \\
\hline Minor $(\mathrm{cm})$ & $2.159 \pm 0.5$ & $2.8 \pm 0.5$ & $2.61 \pm 0.5$ & $2.605 \pm 0.5$ & $4.27 \pm 0.5$ \\
\hline Area $\left(\mathrm{cm}^{2}\right)$ & $4.707 \pm 1.94$ & $8.14 \pm 2.55$ & $7.86 \pm 2.53$ & $7.91 \pm 2.54$ & $14.57 \pm 3.38$ \\
\hline Volume $\left(\mathrm{cm}^{3}\right)$ & $6.78 \pm 4.36$ & $15.19 \pm 7.48$ & $13.66 \pm 7.02$ & $13.75 \pm 7.06$ & $41.43 \pm 14.48$ \\
\hline Accuracy \%* & 24.5 & 54.93 & 49.4 & 49.73 & 149.85 \\
\hline Ratio Major/minor ** & 1.29 & 1.32 & 1.47 & 1.49 & 1.01 \\
\hline
\end{tabular}

*Theoretical Volume was calculate as in (2).

**Values near 1 represent a more spherical shape of ablation zone.

Following the experimental procedure based on Table I, different in-vivo experiments were realized to validate the prototype. The ablation size depend of liver anatomic locations. The presence of heat sinks affects the tissues ablated near the blood vessels. In Fig. 6, it is shown the cross section of thermal lesion for sample of RFA6 test.

Based on successful results, they were chosen the samples of RFA1, RFA3 and RFA6 test. To measure the size of thermal lesion, the ablation zone was approximated to an ellipse (as in (1)), calculating the major diameter, minor diameter and area. See Fig. 6. The Table III summarizes the results for in-vivo experiments.

Table 3: The mean diameters and volumes of the ablated zones according to sample of liver in in-vivo experiments using Boston RF electrode of $4 \mathrm{~cm}$ in diameter.

\begin{tabular}{|c|c|c|c|}
\hline \multirow{2}{*}{ Measures } & \multicolumn{3}{|c|}{ Tests } \\
\cline { 2 - 4 } & RFA 1 & RFA 3 & RFA 6 \\
\hline Major $(\mathrm{cm})$ & $1.49 \pm 0.5$ & $2.54 \pm 0.5$ & $3.76 \pm 0.5$ \\
\hline Minor $(\mathrm{cm})$ & $0.86 \pm 0.5$ & $1.74 \pm 0.5$ & $3.62 \pm 0.5$ \\
\hline Area $\left(\mathrm{cm}^{2}\right)$ & $1.01 \pm 0.93$ & $3.47 \pm 1.68$ & $10.7 \pm 2.9$ \\
\hline${\text { Volume }\left(\mathrm{cm}^{3}\right)}^{\text {Accuracy } \%}{ }^{\mathrm{a}}$ & $0.58 \pm 0.87$ & $4.02 \pm 3.1$ & $25.84 \pm 10.57$ \\
\hline Ratio Major/Minor $^{\mathrm{a} *}$ & 2.11 & 14.52 & 93.5 \\
\hline
\end{tabular}

"Theoretical Volume was calculate as in (2).

${ }^{3 *}$ Values near 1 represent a more spherical shape of ablation zone. 


\section{Discussion}

The aim of this research was to validate the outcomes related to thermal lesion based on ex-vivo and in-vivo experiments using an electromedical prototype for RFA applied to hepatocellular carcinoma. In the case of ex-vivo experiments, the best result was the liver sample number 5, where output power was fixed to $40 \mathrm{~W}$ in whole process achieved a volume of 41.43 $\mathrm{cm}^{3}$ with accuracy percentage of $149.85 \%$ (the size of ablation volume bigger than theoretical volume is accepted but not wanted because it burns healthy tissue. In the case of in-vivo experiments the best result related to volume ablation was RFA6 test, where initial voltage $=10 \mathrm{~V}$, step $=2 \mathrm{~V}$, final voltage $=24 \mathrm{~V}$, total ablation time $=10.8$ minutes achieved a volume of $25.84 \mathrm{~cm}^{3}$ with accuracy percentage of $93.5 \%$ (very good result)), the others accuracy percentage (showed in table 3) are dependent of anatomic location of RF electrode in the liver, for example we verify that the Boston RF electrode was inserted into vessels as suprahepatic vessel, it difficult successful results related to size ablation. In general, the volumes in the ex-vivo experiments are larger than in-vivo experiments, because in the last case the procedure of RFA is affected by heat loss due to perfusion blood (fluid movement). We verified that the ablation protocol and the increase of power causes influence for successful ablation and also it depend of type of electrode, for example with aim to increase the size ablation, if we use RF electrode with cooling system, it is possible increase the size ablation.

The experiments developed in our laboratory, they were done using a power control based on experimental ablation protocol described in the table I. The power used was from $10 \mathrm{~W}$ up to $50 \mathrm{~W}$, frequency of $400 \mathrm{kHz}$ and surgical time between 10 to 20 minutes. The output power used, despite being smaller that commercial equipments (60 W to $200 \mathrm{~W}$ ) supply the burn of tissue and generate tumor necrosis (ablation temperature $>60{ }^{\circ} \mathrm{C}$ ).

\section{Conclusion}

In this research, we proposed an experimental validation system based on ex-vivo and in-vivo experiments for electromedical prototype designed for low-cost and low-power applied in RFA for liver tumors. This work uses several experimental protocol independent of liver anatomic location for analyze the best results related to size thermal lesion. An interesting area of future work for validate our system is development in-vivo experiments with malignant liver tumor (hepatocellular carcinoma), because in this research our results are based on healthy liver.

\section{Acknowledgements}

We'd like to thank to Ministry of Health (MS), Centre for Technological Development (CDT), CNPq Research Productivity Scholarship, Foundation for Research Support (FAP-DF).

\section{References}

[1] D. Haemmerich, "Biophysics of radiofrequency ablation," Critical ReviewsTM in Biomedical Engineering, vol. 38, no. $1,2010$.

[2] B. Zhang, M. Moser, E. Zhang, W. Zhang, "Radiofrequency ablation technique in the treatment of liver tumors: review and future issues," Journal of medical engineering \& technology, vol. 37, no. 2, p. 150159, 2013.

[3] D. Haemmerich, "Mathematical modeling of impedance controlled radiofrequency tumor ablation and ex-vivo validation," in Engineering in Medicine and Biology Society (EMBC), 2010 Annual International Conference of the IEEE, pp. 16051608, 2010.

[4] A. Taheri, P. Mansoori, L. F. Sandoval, S. R. Feldman, D. Pearce, P. M. Williford, "Electrosurgery: part ii. technology, applications, and safety of electrosurgical devices," Journal of the American Academy of Dermatology, vol. 70, no. 4, p. 6071, 2014.

[5] D. M. Overbey, N. T. Townsend, B. C. Chapman, D. T. Bennett, L. S. Foley, A. S.Rau, A. Y. Jeniann, E. L. Jones, G. V. Stiegmann, T. N. Robinson, "Surgical energy-based device injuries and fatalities reported to the food and drug administration," Journal of the American College of Surgeons, vol. 221, no. 1, p. 197205, 2015.

[6] M. J. Ryan, J. Willatt, B. S. Majdalany, A. Z. Kielar, S. Chong, J. A. Ruma, A. Pandya, "Ablation techniques for primary and metastatic liver tumors," World journal of hepatology, vol. 8, no. 3, p. 191, 2016.

[7] C. Lee, E.-K. Choi, H.-J. Kong, Y. B. Choy, H. C. Kim, S. Oh, "Generating radiofrequency ablation lesions using magnetically coupled bipolar catheters," Pacing and Clinical Electrophysiology, vol. 34, no. 8, p. 934938, 2011. 
[8] S. Mulier, Y. Jiang, J. Jamart, C. Wang, Y. Feng, G. Marchal, L. Michel, Y. Ni, "Bipolar radiofrequency ablation with $2 \times 2$ electrodes as a building block for matrix radiofrequency ablation: Ex vivo liver experiments and finite element method modelling," International Journal of Hyperthermia, vol. 31, no. 6, p. 649665, 2015.

[9] A. González-Suárez, M. Trujillo, F. Burdío, A. Andaluz, E. Berjano, "Could the heat sink effect of blood flow inside large vessels protect the vessel wall from thermal damage during rf-assisted surgical resection?," Medical physics, vol. 41 , no. 8, 2014.

[10] R. D. Fonseca, "Deslocamento do Roll Off na Ablação Hepática por Radiofrequência pelo controle da Impedância," M.S.Dissertation, Postgraduate Program of Biomedical Engineering, University of Brasilia, Brasilia, Brazil, 2017.

[11] SCIENTIFIC, B. Radiofrequency Ablation Needle Electrodes. Procedural Algorithms and Lesion Geometry. April 2010 . 